\title{
Publisher's Note: Dynamic model of target charging by short laser pulse interactions
}

[Phys. Rev. E 92, 043107 (2015)]

A. Poyé, J.-L. Dubois, F. Lubrano-Lavaderci, E. D’Humières, M. Bardon, S. Hulin, M. Bailly-Grandvaux, J. Ribolzi, D. Raffestin, J. J. Santos, Ph. Nicolaï, and V. Tikhonchuk

(Received 6 November 2015; published 23 November 2015)

DOI: 10.1103/PhysRevE.92.059902

PACS number(s): 52.38.Kd, 41.20.Gz, 41.75.Jv, 99.10.Fg

This paper was published online on 21 October 2015 with an error in Eqs. (22) and (C10). Equation (22) should read as

$$
\begin{aligned}
\tilde{\phi}_{\mathrm{th}}(z)= & \frac{\lambda+z}{\sqrt{(z+\lambda)^{2}+1}} \ln \left[\frac{\sqrt{(z+\lambda)^{2}+1} \sqrt{z^{2}+1}+1+z(z+\lambda)}{\left(\sqrt{(z+\lambda)^{2}+1}-z-\lambda\right) \lambda}\right] \\
& -\sinh ^{-1}(z)-\ln 2+ \begin{cases}-\ln (\lambda) & \text { if } z<0 \\
-2 \ln (\lambda+z)+\ln (\lambda) & \text { if } \quad z>0\end{cases}
\end{aligned}
$$

Equation (C10) should read as

$$
\begin{aligned}
\tilde{\phi}_{\mathrm{th}}(z)= & \frac{\lambda+z}{\sqrt{(z+\lambda)^{2}+1}} \ln \left[\frac{\sqrt{(z+\lambda)^{2}+1} \sqrt{z^{2}+1}+1+z(z+\lambda)}{\left(\sqrt{(z+\lambda)^{2}+1}-z-\lambda\right) \lambda}\right] \\
& -\sinh ^{-1}(z)-\ln 2+ \begin{cases}-\ln (\lambda) & \text { if } \quad z<0 \\
-2 \ln (\lambda+z)+\ln (\lambda) & \text { if } \quad z>0\end{cases}
\end{aligned}
$$

The equations have been corrected as of 29 October 2015. The equations are correct in the printed version of the journal. 